\title{
3 Research Square

\section{CircTP63 Promotes Cell Proliferation and Invasion by Regulating EZH2 via Sponging miR-217 in Gallbladder Cancer}

\section{Shouhua Wang}

Shanghai Jiaotong University School of Medicine Xinhua Hospital

Huanjun Tong

Shanghai Jiaotong University School of Medicine Xinhua Hospital

Tingting Su

Shanghai Jiaotong University School of Medicine Xinhua Hospital

Di Zhou

Shanghai Jiaotong University School of Medicine Xinhua Hospital

Weibin Shi

Shanghai Jiaotong University School of Medicine Xinhua Hospital

\section{Zhaohui Tang}

Shanghai Jiaotong University School of Medicine Xinhua Hospital

Zhiwei Quan ( $\nabla$ quanzhiwei@xinhuamed.com.cn )

https://orcid.org/0000-0002-9317-1583

\section{Research article}

Keywords: Gallbladder cancer (GBC), CircRNA TP63, qRT-PCR, miR-217, tumor, therapeutic strategy

Posted Date: July 13th, 2021

DOI: https://doi.org/10.21203/rs.3.rs-553740/v2

License: (c) (i) This work is licensed under a Creative Commons Attribution 4.0 International License.

Read Full License

Version of Record: A version of this preprint was published at Cancer Cell International on November 17th, 2021. See the published version at https://doi.org/10.1186/s12935-021-02316-w. 


\section{Abstract}

Background: Gallbladder cancer (GBC) is the most common biliary tract malignancy and has a poor prognosis in patients with GBC. CircRNA TP63 (circTP63) has been implicated in some tumor proliferation and invasion in some tumors. The study aims to investigate the clinical significance and functional role of circTP63 in GBC.

Methods: The expression of circTP63 in GBC was detected by qRT-PCR and the association between circTP63 expression and prognosis of GBC patients was analyzed. CCK8 assay, flow cytometry analysis, transwell assay and in vivo studies were used to evaluated the cell proliferation and invasion after circTP63 knockdown in GBC cells. Luciferase reporter assays and RNA pull-down assay were used to determine the correlation between circTP63 and miR-217. Besides, western blot analysis was also performed.

Results: In the present study, we showed that circTP63 expression was upregulated in GBC tissues and cells. Higher circTP63 expression was associated with lymph node metastasis and short overall survival (OS) in patients with GBC. In vitro, knockdown of circTP63 inhibited cell proliferation, cell cycle progression, migration and invasion in GBC. Besides, we demonstrated that knockdown of circTP63 inhibited GBC cell EMT process. In vivo, knockdown of circTP63 inhibited tumor growth in GBC. Mechanistically, we demonstrated that circTP63 competitively bind to miR-217 and promoted EZH2 expression and finally facilitated tumor progression.

Conclusions: Our findings demonstrated that circTP63 sponge miR-217 and regulated EZH2 expression and finally facilitates tumor progression. Thus, targeting circTP63 may be a therapeutic strategy for the treatment of GBC.

\section{Introduction}

Gallbladder cancer (GBC) is one of the most common digestive tract tumors worldwide [1]. GBC is characteristically diagnosed at advanced stages due to the absence of specific signs and symptoms, and only a small population of GBC patients is suitable for the surgical resection, contributing to the worse 5years overall survival rate [2-3]. Elucidating the mechanism of the occurrence and development of GBC and validating existing novel molecular biomarkers to improve patient outcomes are needed.

Circular RNAs (circRNAs) are a type of endogenous non-coding RNA that do not have a 5'-cap or a 3'polyA tail and are implicated in a variety of biological functions [4-5]. Recently, RNA-sequences revealed that circRNAsare involved in diagnosis, prognosis, development, and drug resistance in tumors. CircRNAswere found to regulate cell apoptosis, cell proliferation, cell migration and tumor metastasis by regulating gene expression [6].Such as, hsa_circRNA_100269 expression is downregulated in gastric cancer and upregulated hsa_circRNA_100269 inhibits cell growth and tumor metastasis through inactivating PI3K/Akt axis [7].Up-regulated circBACH2 is found in triple-negative breast cancer and contributes to cell proliferation, invasion, and migration of triple-negative breast cancer[8].circCCDC66 
expression is upregulated in thyroid cancer and promotes cell proliferation, migratory and invasive abilities and glycolysis through the miR-211-5p/PDK4 axis[9].Hsa_circ_0068515, designated as circTP63, is reported inlung squamous cell carcinoma (LUSC) and correlates with larger tumor size and higher TNM stage in LUSC patients. Besides, upregulated circTP63is also identified to promote cell proliferation by functioning as a ceRNA to upregulate FOXM1 in LUSC [10].Another study shows that circular RNA circTP63 enhances estrogen receptor-positive breast cancer progression and malignant behaviors through the miR-873-3p/FOXM1 axis [11].In hepatocellular carcinoma (HCC), circTP63 is significantly upregulated in HCC tissues and cell lines and circTP63 overexpression promotes tumor progression by sponging miR-155-5p and upregulating ZBTB18 expression [12]. However, its role in GBC progression remains unknown.

In the study, we firstly demonstrated that circTP63 expression was significantly upregulated in GBCtissues and cells. Upregulated circTP63 expression notably associated with short survival rate of GBC patients. Functionally, knockdown of circTP63 inhibited cell proliferation, cell cycle progression, migration and invasion abilities in GBC and suppressed tumor growth in vivo. Besides, we demonstrated that knockdown of circTP63 inhibited cell EMT process in GBC. Mechanistically, we showed that circTP63 could competitively bind to miR-217 and promoted level of EZH2 expression and finally facilitated tumor progression. Thus, these results provided better understand for the regulatory role of circRNAs in GBC progression, and could improve the diagnosis and therapies of GBC.

\section{Materials And Methods}

\section{Patients tissue samples}

The study was performed in accordance with the Declaration of Helsinki and the guidelines of the committee of the Human Ethics Committee of Xinhua Hospital. A total of 39 snap-frozen GBC tissues and paired adjacent normal tissues were acquired from patients diagnosed with GBC at Xinhua Hospital.All the enrolled patients of this study had never received preoperative therapy and tissue samples were collected and frozen in liquid nitrogen immediately after surgical resection. The patient information was shown in Table 1. All of participants signed informed consent before this study.

\section{Cell lines culture}

Three human GBC cell lines (NOZ, GBC-SD, and SGC-996) and the human intrahepatic biliary epithelialcell line HIBEC used in the present studywere purchased from Cell Bank of theChinese Academy of Science (Shanghai, China). Cells were cultured in DMEM (Gibco, Invitrogen, USA) supplemented with $10 \%$ fetal bovine serum (FBS) (Gibco, Invitrogen, US, lot: 10099-141c) and 0.5\% penicillin/streptomycin (Gibco, Invitrogen, USA). Cells were maintained at $37^{\circ} \mathrm{C}$ in a humidified atmosphere containing $5 \% \mathrm{CO}_{2}$.

\section{Cell transfection}


Cell transfection was performed with Lipofectamine 2000 or 3000 Reagent (Invitrogen, CA, USA) according to the manufacturer's protocol. The siRNA sequences for transfection were purchased from GenePharma (Shanghai, China).The sequences for circTP63 was as follows:si-circTP63-1, 5'GCCAACAGUGAGGGGCCGU-3';si-circTP63-2,5'-CAACAGUGAGGGGCCGUGAGA-3';siRNAcontrol(si-NC)5'UUCUCCGAACGUGUCACGU-3'.MiR-217mimic, miR-217 inhibitor and miR-NC were obtained from Gene Pharma (Shanghai, China).

\section{CCK8 assays}

Transfected NOZ and SGC-996 cells were seeded in 96-well plates $\left(3 \times 10^{3}\right.$ cells per well). After $2 \mathrm{~h}$, cells were added with $10 \mu$ l of the CCK-8 solution (Dojindo Laboratories, Kumamoto, Japan) in each well of the plate. Then,cells were incubated for $2 \mathrm{~h}$ in the incubator. Finally, the absorbance was detected at $450 \mathrm{~nm}$ using a microplate reader (BioTek Instruments, Inc., Winooski, VT, USA).

\section{Flow cytometry analysis}

Transfected cells were harvested, washed and then were fixed with $70 \%$ ethanol at $-20^{\circ} \mathrm{C}$ overnight, Next, after RNase digestion, the cells were stained with $20 \mu \mathrm{g} / \mathrm{ml}$ Propidium iodide (Pl; Beyotime, Shanghai, China) at $37^{\circ} \mathrm{C}$ for $30 \mathrm{~min}$, and $100 \mu \mathrm{g} / \mathrm{ml}$ RNase A was subsequently added to the cells and incubated in a $4{ }^{\circ} \mathrm{C}$ dark room for 30 min.Cell cycle was examined by flow cytometry using the FACS Calibur system (BD Biosciences, San Jose, CA, USA).

\section{Cell migration and invasion assays}

Cell migration or invasion assays were performed by transwell plates (BD Falcon, USA) and were coated without or without Matrigel in 24-well transwell chambers with $8 \mathrm{~mm}$ pore polycarbonate filters (Millipore, Billerica, MA, USA). $1 \times 10^{5}$ cells were cultured on the upper chamber in medium without serum, while the lower chamber was added with $10 \%$ fetal bovine serum (FBS) (Gibco, Invitrogen, USA). After transfection at 48 hours, cells on the lower layer of the membrane were stained using 1\% crystal violet for $30 \mathrm{~min}$ at room temperature. The cell number was counted by using an Olympus microscopeand five fields were randomly selected to count the cells(Magnification 200X). All assays were independently performed in triplicate.

\section{Quantitative Real-Time PCR (QRT-PCR) analysis}

Total RNA was extracted from tissues or cells usingTRIzol reagent (Invitrogen). The cDNA was synthesized using a Prime Script RT reagent kit (Takara, Japan). The mRNA expression was analyzed by using SYBR Green Real-Time PCR Master Mixes (Thermo Fisher Scientific, USA) by an ABI 7900 Fast Thermal Cycler (Applied Biosystems; Thermo Fisher Scientific, USA). The GAPDH or U6 was used as reference. The primer sequences were as follow: the circTP63 forward: $5^{\prime}$-GCCCTCACTCCTACAACCATT3';reverse:5'-TTGTGTGCTGAGGAAGGTACT-3';TheEZH2-forward:5'-TGCAGTTGCTTCAGTACCCATAAT3';EZH2-reverse:5'-ATCCCCGTGTACTTTCCCATCATAAT-3';GAPDH-forward:5'-AAGGTGAAGGTCGGAGTCA- 
3';GAPDH-reverse:5'-GGAAGATGGTGATGGGATTT-3';U6-forward:5'-CTCGCTTCGGCAGCACA-3';U6-

reverse:5'-AACGCTTCACGAATTTGCGT-3'. The relative mRNA expression was calculated using the $2^{-\triangle \triangle C t}$ methods.

\section{Western blotting assays}

Total protein was extracted using a RIPA buffer (Beyotime, Beijing, China). An equal amount of total protein was separated on SDS-polyacrylamide gel electrophoresis (SDS-PAGE) and then transferred onto PVDF membranes (Millipore, Billerica. MA. USA). The membrane was blocked with $5 \%$ non-fat milk and incubated with the primary antibody with E-cadherin (1:1000, Cell Signaling Technology, Houston, TX, USA). Vimentin (1:1000, Cell Signaling Technology, Houston, TX, USA), EZH2 (1:1000, Cell Signaling Technology, Houston, TX, USA) and GAPDH (1:2000, Abcam) overnight at $4{ }^{\circ} \mathrm{C}$. Next, the secondary antibodies were added for 1.5 hours and then each protein band was detected by the ECL detection system (Amersham Biosciences, Buckinghamshire, UK).

\section{Luciferase reporter assays}

The wide type (WT)circPVT1 or EZH2 3'-untranslated region (UTR) containing miR-217 targeting sequenceand the mutated type (MUT) was amplified and cloned into the luciferase reporter plasmid psicheck-2 vector (Promega, Madison, WI). Cells were collected and lysed for luciferase detection 48 hours after transfection. Luciferase activities were measured using the Dual-Luciferase Reporter Assay System (Promega, USA). The relative luciferase activity was normalized against to the Renilla luciferase activity.

\section{Biotin-coupled RNA pulldown}

The 3'end biotinylated miR-217 mimic or control RNA was designed and synthesized by Genepharm (Suzhou, China).NOZ were transfected with $50 \mathrm{nM}$ of biotin-labeled miRNAs (Gene Create, Wuhan, China). Streptavidin-coupled Dynabeads (Invitrogen) were washed, resuspended in the buffer and then was added with the biotin-labeled miRNAs. After incubating at room temperature for $10 \mathrm{~min}$, the coated beads were separated with a magnet for 2 min. The pulled-down RNA was extracted by Trizol reagent and followed by qRT-PCR analysis.

\section{In vivo xenograft experiments}

Xenograft experiments ( $n=5 /$ per group) were performed by using 3-week BALB/c nude mice. All animal protocols were approved by the Institutional Animal Care and Use Committee at Xinhua Hospital. $1 \times 10^{5}$ NOZ cells were transfected and were subcutaneously injected into the flank. The tumor volume and weight were evaluated every week, Tumor volume $(\mathrm{mm} 3)=($ length $) \times(\text { width })^{2} / 2$. After 4 weeks, mice were sacrificed and tissues processed for further histological analysis.According to the AVMA Guidelines for the Euthanasia of Animals, all the mice were euthanized with an intraperitoneal injection of a three-fold 
dose of barbiturates. After that we removed tumors immediately and measured the length, width and weight of the tumors. No mice died accidentally during feeding.

\section{Statistical analysis}

All statistical analyses were performed using GraphPad Prism (GraphPad Software, Inc. La Jolla, USA). Statistical analysis was carried out using $t$ test or Bonferroni Multiple Comparisons Test, A $p$ value of less than 0.05 was considered statistically significant.

\section{Results}

\section{CircTP63 expression is upregulated in GBC and correlates with poor prognosis}

To explore the clinical significance of circTP63 in GBC, we detected the circTP63 expression collected from 39 pairs of GBC tissues and adjacent normal tissues by qRT-PCR. The results showed that circTP63 expression was dramatically upregulated compared with adjacent normal tissues in patients with GBC (Figure 1A). Moreover, we also detected that circTP63 expression was higher in three GBC cell lines than in HIBEC cells (Figure 1B). The 39 cases of patients were divided into circTP63 higher expression and lower expression groups according to the medium value. The association between circTP63 expression and clinicopathological characteristics was analyzed.The results displayed that circTP63 expression was associated with lymph node metastasis (Table 1, P<0.05). Kaplan-Meier analyses and log rank test showed that higher circTP63 expression showed a poor overall survival rate in GBC patients compared with lower circTP63 expression (Figure 1C). We next examined the subcellular localization of circTP63, and the results demonstrated that circTP63 expression was predominately distributed in the cytoplasm than that in the nucleus in NOZ and SGC-996 cells (Figure1D-E). Total RNA isolated from NOZ and SGC996 cells was exposed to RNase R and the results presented that circTP63 expression had no apparent change after RNase R treatment (Figure1F).

\section{Downregulation of circTP63 inhibits cell proliferation, migration and invasion in GBC}

Two specific circTP63 siRNAs were used to knock down the expression of circTP63 in GBC cells. The qRTPCR analysis results showed that the expression level of circTP63 was markedly reduced in NOZ and SGC-996 cells (Figure 2A).Cell proliferation was assessed by CCK8 assay, and the results showed that knockdown of circTP63suppressed cellproliferation ability compared the control group in NOZ and SGC996 cells (Figure 2B-2C). In addition, flow cytometry analysis also demonstrated that circTP63 silencing inhibited S phase cell number but increased G1 cell number compared the control group in NOZ and SGC996 cells (Figure 2D-2G).Moreover, transwell assay was performed to detect cell migration and invasion ability, and the results showed that the cell migration and invasion ability were impaired by circTP63 silencing compared the control group in NOZ and SGC-996 cells (Figure 3A-3D). The epithelial marker Ecadherin expression was notably increased, while the expression of mesenchymal marker Vimentin was notably decreased in NOZ and SGC-996 cells, after cells were transfected with si-circTP63-1 or si- 
circTP63-2 compared the control group(Figure 3E-3F). These above evidences showed that circTP63 downregulation suppressed GBC cell growth and EMT process.

\section{Downregulation of circTP63 inhibits cell growth in vivo in GBC}

To evaluate the roles of circTP63 expression in vivo, we constructed xenograft tumors in nude mice by injection with NOZ cells stably expressing sh-circTP63 or control vector by using lentiviral transduction. The results showed that the sh-circTP63 group had slower growth rate and tumor weigh than those expressing control vector (Figure 4A-4C). Immunohistochemistry staining of Ki-67 expression in xenograft tumors demonstrated that tumor tissues in sh-circTP63 group had less Ki-67 positive cells than that in the control group (Figure 4D). These results suggested that circTP63 knockdown could inhibit tumor growth in vivo.

\section{circTP63 sponges miR-217 in GBC cells}

Recently, more studies have reported that circRNAs could sponging to miRNAs, thereby reducing the regulation of miRNAs on their target genes[13].By performing a search for miRNAs that have complementary base pairing with circTP63 by using the online software tools circinteractome (http://circinteractome.nia.nih.gov), the results showed that miR-217 could form complementary base pairing with circTP63 (Figure 5A).Luciferase assay demonstrated that miR-217 mimic repressed the luciferase activity of circTP63-WT, while miR-217 mimic had little effect on that of circTP63-MUT in NOZ cells (Figure 5B). We then detected the expression of miR-217 after knockdown of circTP63, the results indicated that miR-217 expression was upregulated after knockdown of circTP63 in NOZ and SGC-996 cells compared with the control group (Figure 5C). Furthermore, we also detected that miR-217 expression was significantly lower in GBC tissues compared with adjacent normal tissues by qRT-PCR analysis (Figure 5D). The miR-217 expression is also lower expression in three GBC cell lines than in HIBEC cells (Figure 5E).In addition, we demonstrated that circTP63 were pulled down and enriched with 3'-end biotinylated miR-217 in NOZ cells compared with the control group(Figure 5F).

\section{CircTP63 sponges miR-217 and regulates EZH2 expression in GBC}

It was reported that the miR-217 could regulate the EZH2 expression in gallbladder cancer [14], we sought to explore whether circTP63 expression could affect EZH2expression.The EZH2expression was significantly higher in GBC tissues compared with adjacent normal tissues by qRT-PCR analysis(Figure 6A).EZH2 was predicted as a target gene of miR-217(Figure 6B). EZH2 was notably decreased in NOZ and SGC-996 cells transfected with si-circTP63-1 or si-circTP63-2 compared the control group (Figure 6C). Luciferase assays demonstrated that miR-217 mimic repressed the luciferase activity of EZH2-WT, while miR-217 mimic had little effect on that of EZH2-MUT in NOZ cells (Figure 6D). We also demonstrated that EZH2 mRNA expression was downregulated in NOZ and SGC-996 after circTP63 knockdown, but was rescued by transfecting with miR-217 inhibitor and si-circTP63-1(Figure 6E-6F). The western blot analysis showed that EZH2 protein expression was downregulated in NOZ and SGC-996 cells transfected with sicircTP63-1 and si-circTP63-2, but was rescued by transfected miR-217 inhibitor and si-circTP63-1(Figure 
6G-6H). Thus, these results indicated that circTP63 sponged miR-217 and regulated EZH2 expression in GBC.In our previous study, we demonstrated that EZH2 is upregulated in GBCand is a key downstream target of IncRNA MINCR, which regulates cell proliferation, cell invasive and apoptosis in GBC cells[15]. In

the study, we revealed a novel regulatory pathway that circTP63 sponged miR-217 and regulated EZH2 expression in GBC.

\section{Discussion}

Gallbladder carcinoma presents a high degree of malignancy and extremely dismal prognosis for patients. Recently, increasing studiesare beginning to explore the new therapy methodsderiving from molecular mechanismsfor GBC [16]. Accumulating evidence regarding the multifunctionalities of circRNAs make them ideal for targets and markers for the prognosis, diagnosis, and developing new treatments of cancer [17]. Such as, circular RNA circ-MTO1 expression is upregulated in GBC tissues and serves as a novel potential diagnostic and prognostic biomarker for gallbladder cancer[18]. Huang et al reported that circular RNA circERBB2 promotes gallbladder cancer proliferation in vitro and in vivo. Furthermore, circERBB2 regulates nucleolar localization of PA2G4, thereby forming a circERBB2-PA2G4TIFIA regulatory axis to modulate ribosomal DNA transcription and GBC proliferation [19]. Our previous study reported that by RNA sequencing from GBC tissues, circFOXP1 promoted GBC progression and Warburg effect by regulating PKLR expression, suggesting a potential target for GBC treatment [20]. However, the study for circTP63 in GBC progression remains less.

In the study, our results demonstrated that circTP63 expression was upregulated in GBC tissues compared to adjacent normal tissues. We also detected that circTP63 expression is higher in GBC cell lines. Furthermore, clinical data by K-M analysis and log rank test showed that higher circTP63 expression showed a poor overall survival rate in GBC patients compared with lower circTP63 expression. These clinical results indicated that circTP63 expression could be a prognostic maker for GBC.

In the previous study, CircTP63 are identified as vital regulators in several tumors. Such as, circTP63 expression is elevated in lung squamous cell carcinoma (LUSC) tissues and is correlated with larger tumor size and higher TNM stage in LUSC patients. Function studies showed circTP63 promotes cell proliferation both in vitro and in vivo by competitively binding to miR-873-3p and regulating the level of FOXM1 [10]. In breast cancer, Deng et al found that circular RNA circTP63 enhances estrogen receptorpositive breast cancer progression and malignant behaviors through the miR-873-3p/FOXM1 axis [11]. Our results showed that circTP63 knockdown inhibited cell proliferation and cell cycle progression. Furthermore, we demonstrated that circTP63 knockdown inhibited cell migration, cell invasion ability and EMT process. These evidences indicated that circTP63 affected GBC cell growth and EMT process.

Next, we explored the underlying molecular mechanisms of circTP63 in GBC. CircRNAs were reported to exert their functions such as 'microRNA sponge' that competitively bound to miRNAs. In the study,we performed bioinformatic analyses to select miRNAs, which shared common binding sites with circTP63. The results showed that miR-217 shared common binding sites with circTP63. Simultaneously, we found 
that miR-217reduced the luciferase activity of circTP63 luciferase reporter compared to control.After circTP63 knockdown, the miR-217 expression was upregulated in GBC cells. In addition, we demonstrated that circTP63 were pulled down and enriched with 3'-end biotinylated miR-217 in GBC cells compared with the control group, these results indicated that circTP63 could interacted with miR-217 in GBC.

EZH2is found to be upregulated in GBC tissues in previous study and overexpression of EZH2 is associated with invasion, metastasis, and poor progression of gallbladder adenocarcinoma [21].Long noncoding RNA MEG3 regulates LATS2 by promoting the ubiquitination of EZH2 and inhibits proliferation and invasion in gallbladder cancer[22]. Our previous study showed that EZH2 expression is also higher in GBC tissues and enhanced GBC tumor progression, then, we showed that IncRNA MINCR/miR-26a5p/EZH2 axis was involved in cell proliferation, cell invasive and apoptosis in GBC cells [23]. In the study, we found a novel regulating pathway that circTP63/miR-217/EZH2 affected GBC cell proliferation and invasion.

\section{Conclusions}

In conclusion, our study firstly explored the biological significance of circTP63 in GBC. We demonstrated that circTP63 expression was significantly upregulated in GBC tissues. Higher circTP63 predicted the poor prognosis of GBC patients. CircTP63 downregulation inhibited the GBC cells proliferation and metastasis. Moreover, we found that circTP63 knockdown inhibited EZH2expression by sponging to miR217. Therefore, circTP63 inhibition might serve as a potential therapeutic target for GBC patients.

\section{Abbreviations}

GBC, Gallbladder cancer; OS, overall survival; circRNAs, circular RNAs; cDNA: complementary DNA; qRTPCR, quantitative real-time PCR; RIP, RNA immunoprecipitation; SDS/PAGE, SDS-polyacrylamide gel electrophoresis; PCNA, proliferating cell nuclear antigen; WT, wild-type; MUT, mutant-type; ceRNA, competing endogenous RNA

\section{Declarations}

\section{Acknowledgments}

None.

\section{Consent for publication}

Not applicable.

\section{Competing interests}

The authors declare that they have no competing interests. 


\section{Authors' contributions}

ShouhuaWang,Zhaohui Tang and ZhiweiQuanconceived the project and supervised the project. Di Zhou,ShouhuaWang and HuanjunTong performed the biological experiments, TingtingSu, Di Zhou, Weibin Shi, and Zhiwei Quananalyzed data and wrote the manuscript. Both authors read and approved the final manuscript.

\section{Funding}

This work was supported by the National Natural Science Foundation of China (grant number 81572297 and 81802315) and Sponsored by Shanghai Sailing Program (grant number18YF1416000).

\section{Availability of data and materials}

The datasets used and/or analyzed during the current study are available from the corresponding author on reasonable request.

\section{Ethics approval and consent to participate}

The present study was approved by the ethics committee ofXinhua Hospital.Written informed consent was obtained from all patients and conducted in accordance with the Declaration of Helsinki.

\section{References}

1. Huang YP, Liu K, Wang YX, Yang Y, Xiong L, Zhang ZJ, Wen Y. Application and research progress of organoids in cholangiocarcinoma and gallbladder carcinoma. Am J Cancer Res. 2021;11(1):31-42.

2. Valle JW, Kelley RK, Nervi B, Oh DY, Zhu AX. Biliary tract cancer. Lancet. 2021; 397(10272):428-444.

3. Song X, Hu Y, Li Y, Shao R, Liu F, Liu Y. Overview of current targeted therapy in gallbladder cancer. Signal Transduct Target Ther. 2020;5(1):230.

4. Turai PI, Nyírő G, Butz H, Patócs A, Igaz P. MicroRNAs, Long Non-Coding RNAs, and Circular RNAs: Potential Biomarkers and Therapeutic Targets in Pheochromocytoma/Paraganglioma. Cancers (Basel). 2021;13(7):1522.

5. Li J, Xu Q, Huang ZJ, Mao N, Lin ZT, Cheng L, Sun B, Wang G. CircRNAs: a new target for the diagnosis and treatment of digestive system neoplasms. Cell Death Dis. 2021;12(2):205.

6. Wang X, Li H, Lu Y, Cheng L. Circular RNAs in Human Cancer. Front Oncol. 2021; 10:577118.

7. Wang Z, Liu C. Upregulated hsa_circRNA_100269 inhibits the growth and metastasis of gastric cancer through inactivating PI3K/Akt axis. PLoS One. 2021;16(4): e0250603.

8. Wang X, Xue B, Zhang Y, Guo G, Duan X, Dou D. Up-regulated circBACH2 contributes to cell proliferation, invasion, and migration of triple-negative breast cancer. Cell Death Dis. 2021;12(5):412.

9. Ren H, Song Z, Chao C, Mao W. circCCDC66 promotes thyroid cancer cell proliferation, migratory and invasive abilities and glycolysis through the miR-211-5p/PDK4 axis. Oncol Lett. 2021;21(5):416. 
10. Cheng Z, Yu C, Cui S, Wang H, Jin H, Wang C, Li B, Qin M, Yang C, He J, Zuo Q, Wang S, Liu J, Ye W, Lv $Y$, Zhao F, Yao M, Jiang L, Qin W. circTP63 functions as a ceRNA to promote lung squamous cell carcinoma progression by upregulating FOXM1. Nat Commun. 2019;10(1):3200.

11. Deng Y, Xia J, Xu YE. Circular RNA circTP63 enhances estrogen receptor-positive breast cancer progression and malignant behaviors through the miR-873-3p/FOXM1 axis. Anticancer Drugs. 2021;32(1):44-52.

12. Wang J, Che J. CircTP63 promotes hepatocellular carcinoma progression by sponging miR-155-5p and upregulating ZBTB18. Cancer Cell Int. 2021;21(1):156.

13. Han C, Seebacher NA, Hornicek FJ, Kan Q, Duan Z. Regulation of microRNAs function by circular RNAs in human cancer. Oncotarget. 2017;8(38):64622-64637.

14. Chen DL, Zhang DS, Lu YX, Chen LZ, Zeng ZL, He MM, Wang FH, Li YH, Zhang HZ, Pelicano H, Zhang W, Xu RH. microRNA-217 inhibits tumor progression and metastasis by downregulating EZH2 and predicts favorable prognosis in gastric cancer. Oncotarget. 2015;6(13):10868-79.

15. Wang SH, Yang Y, Wu XC, Zhang MD, Weng MZ, Zhou D, Wang JD, Quan ZW. Long non-coding RNA MINCR promotes gallbladder cancer progression through stimulating EZH2 expression. Cancer Lett. 2016;380(1):122-33.

16. Verduci L, Tarcitano E, Strano S, Yarden Y, Blandino G. CircRNAs: role in human diseases and potential use as biomarkers. Cell Death Dis. 2021;12(5):468.

17. Brozzi F, Regazzi R. Circular RNAs as Novel Regulators of $\beta$-Cell Functions under Physiological and Pathological Conditions. Int J Mol Sci. 2021;22(4):1503.

18. Wang X, Lin YK, Lu ZL, Li J. Circular RNA circ-MTO1 serves as a novel potential diagnostic and prognostic biomarker for gallbladder cancer. Eur Rev Med Pharmacol Sci. 2020 Aug;24(16):83598366.

19. Huang X, He M, Huang S, Lin R, Zhan M, Yang D, Shen H, Xu S, Cheng W, Yu J, Qiu Z, Wang J. Circular RNA circERBB2 promotes gallbladder cancer progression by regulating PA2G4-dependent rDNA transcription. Mol Cancer. 2019;18(1):166.

20. Wang S, Zhang Y, Cai Q, Ma M, Jin LY, Weng M, Zhou D, Tang Z, Wang JD, Quan Z. Circular RNA FOXP1 promotes tumor progression and Warburg effect in gallbladder cancer by regulating PKLR expression. Mol Cancer. 2019;18(1):145.

21. Liu DC, Yang ZL. Overexpression of EZH2 and loss of expression of PTEN is associated with invasion, metastasis, and poor progression of gallbladder adenocarcinoma. Pathol Res Pract. 2011;207(8):472-8.

22. Jin L, Cai Q, Wang S, Wang S, Mondal T, Wang J, Quan Z. Long noncoding RNA MEG3 regulates LATS2 by promoting the ubiquitination of EZH2 and inhibits proliferation and invasion in gallbladder cancer. Cell Death Dis. 2018;9(10):1017.

23. Wang SH, Yang Y, Wu XC, Zhang MD, Weng MZ, Zhou D, Wang JD, Quan ZW. Long non-coding RNA MINCR promotes gallbladder cancer progression through stimulating EZH2 expression. Cancer Lett. 2016;380(1):122-33. 


\section{Tables}

Table 1 The association between circTP63 expression and clinicopathological factors in GBC patients.

\section{CircTP63 expression}

\begin{tabular}{|c|c|c|c|c|}
\hline Clinicopathological factors & $\begin{array}{l}\text { The number of patients } \\
(n=39)\end{array}$ & $\begin{array}{l}\text { Lower } \\
(n=19)\end{array}$ & $\begin{array}{l}\text { Higher } \\
(\mathrm{n}=20)\end{array}$ & P-value \\
\hline Age & & & & 0.839 \\
\hline$\leq 60$ & 24 & 12 & 12 & \\
\hline$>60$ & 15 & 7 & 8 & \\
\hline Gender & & & & 0.798 \\
\hline Male & 11 & 5 & 6 & \\
\hline Female & 28 & 14 & 14 & \\
\hline Tumor size & & & & 0.894 \\
\hline$<5 \mathrm{~cm}$ & 16 & 8 & 8 & \\
\hline$\geq 5 \mathrm{~cm}$ & 23 & 11 & 12 & \\
\hline Histological grade & & & & 0.429 \\
\hline well and moderately & 21 & 9 & 12 & \\
\hline Poorly and others & 18 & 10 & 8 & \\
\hline \multicolumn{5}{|l|}{ Lymph node metastasis } \\
\hline & & & & $0.038^{*}$ \\
\hline Negative & 18 & 12 & 6 & \\
\hline Positive & 21 & 7 & 14 & \\
\hline Adjacent organ invasion & & & & 0.511 \\
\hline No & 16 & 9 & 7 & \\
\hline Yes & 23 & 10 & 12 & \\
\hline TNM stage & & & & 0.264 \\
\hline$|-I|$ & 19 & 11 & 8 & \\
\hline III-IV & 20 & 8 & 12 & \\
\hline
\end{tabular}

${ }^{*} \mathrm{P}<$ 0.05.TNM, tumor-node-metastasis. 
A

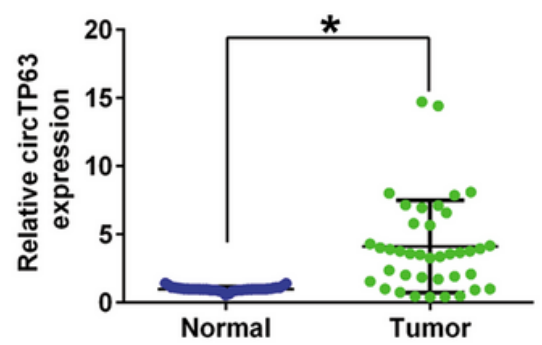

D

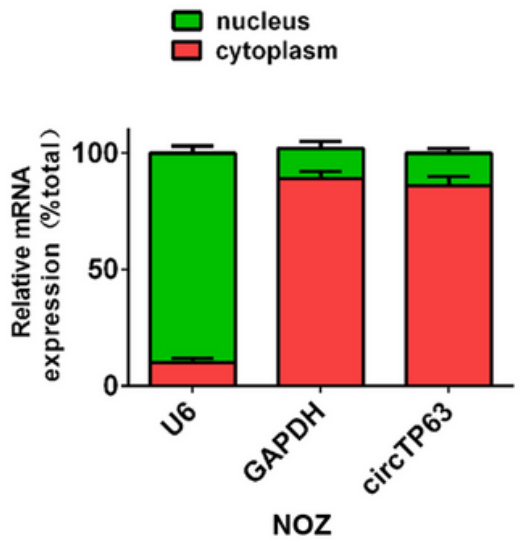

B

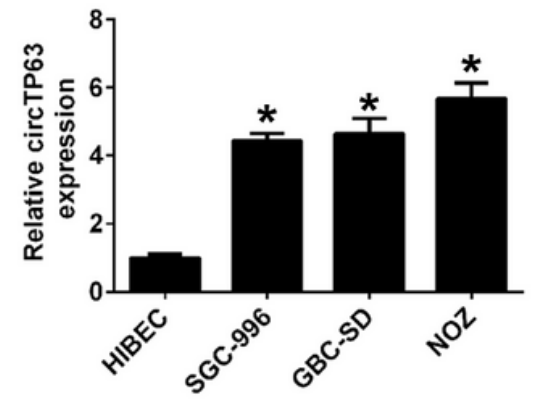

$E$

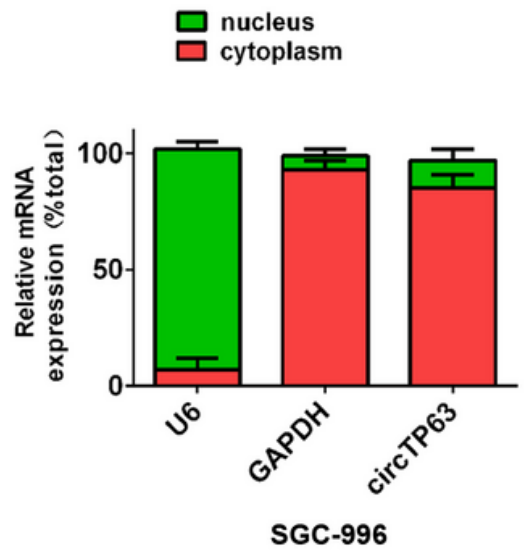

C

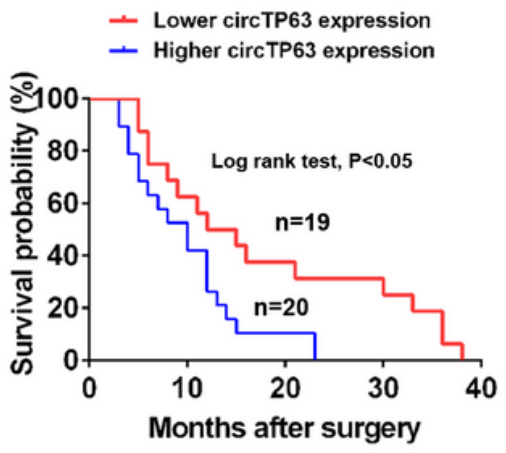

$\mathrm{F}$

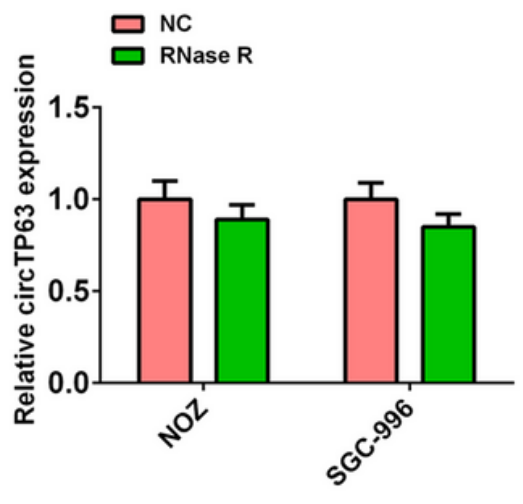

Figure 1

CircTP63 expression is upregulated in GBC. (A) The relative expression of circTP63 was detected in GBC tissues and adjacent normal tissues by qRT-PCR analysis. (B) The relative expression of circTP63 was detected in three GBC cell lines (NOZ, SGC-996 and GBC) and the human intrahepatic biliary epithelial cell line HIBEC by qRT-PCR analysis. (C) Kaplan-Meier survival curves indicating the correlation between circTP63 and overall survival in GBC patients. (D)-(E) Nucleocytoplasmic fractionation experiment demonstrated that circTP63 expression was mainly distributed in the cytoplasm. (F) QRT-PCR was performed to detect the circTP63 expression from control or digested RNAs using RNase R exonuclease in NOZ and SGC-996 cells. Data are shown as mean \pm S.E.M., ${ }^{*} P<0.05$. 
A

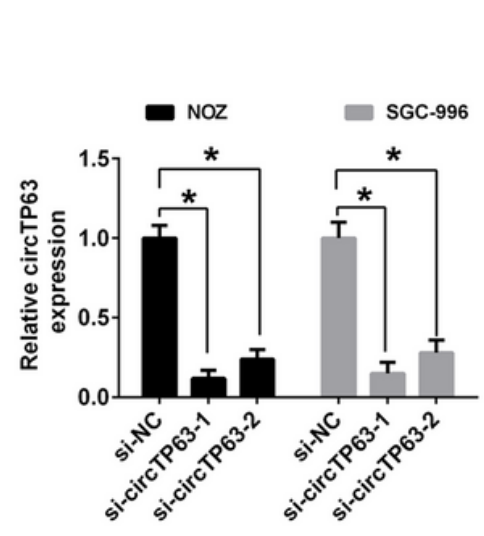

B

$\underset{\text { Ni-NC }}{\mathrm{NOZ}}$ si-circTP63-1 - si-circTP63-2

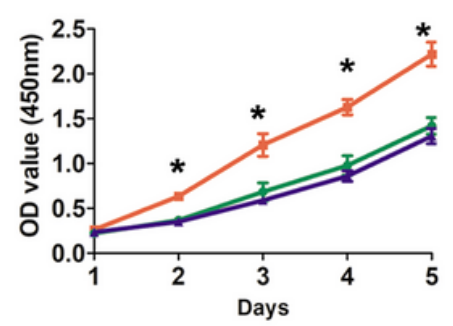

D

si-NC

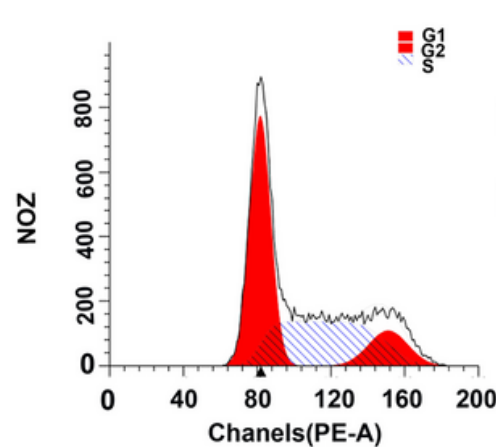

F

si-NC

si-circTP63-1
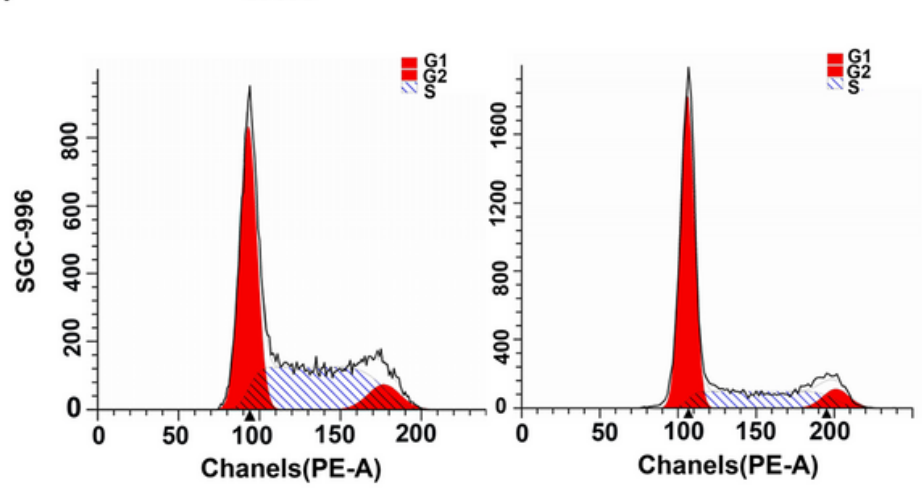

C

SGC-996

$=$ si-NC $\rightarrow$ si-circTP63-1 - si-circTP63-2

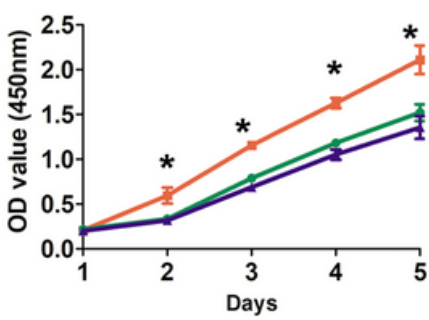

E

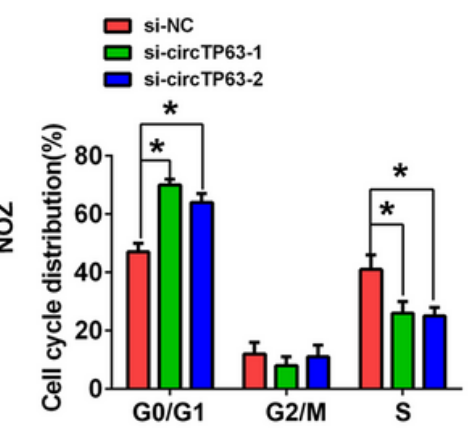

G

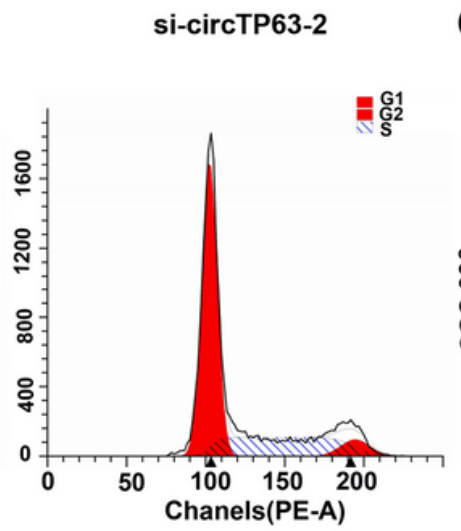

$\square$ si-NC

$\square$ si-circTP63-1

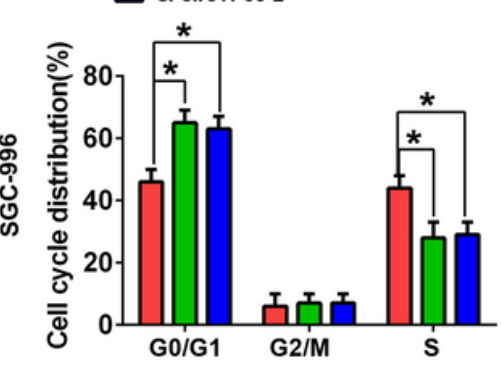

Figure 2

CircTP63 knockdown suppressed cell proliferation and cell cycle progress in GBC. (A) The relative expression of circTP63 was detected in NOZ and SGC-996 cells by qRT-PCR analysis after cells were transfected with si-NC, si-circTP63-1 or si-circTP63-2. (B)-(C) The cell proliferation ability was evaluated by CCK8 assays in NOZ and SGC-996 cells after cells were transfected with si-NC, si-circTP63-1 or sicircTP63-2 at 1, 2,3,4 and 5 days. (D)-(E) The cell cycle progression was analyzed by flow cytometry after cells were transfected with si-NC, si-circTP63-1 or si-circTP63-2 in NOZ cells. (F)-(G) The cell cycle progression was analyzed by flow cytometry after cells were transfected with si-NC, si-circTP63-1 or sicircTP63-2 in SGC-996 cells. Data are shown as mean \pm S.E.M., ${ }^{*} P<0.05$. 
A

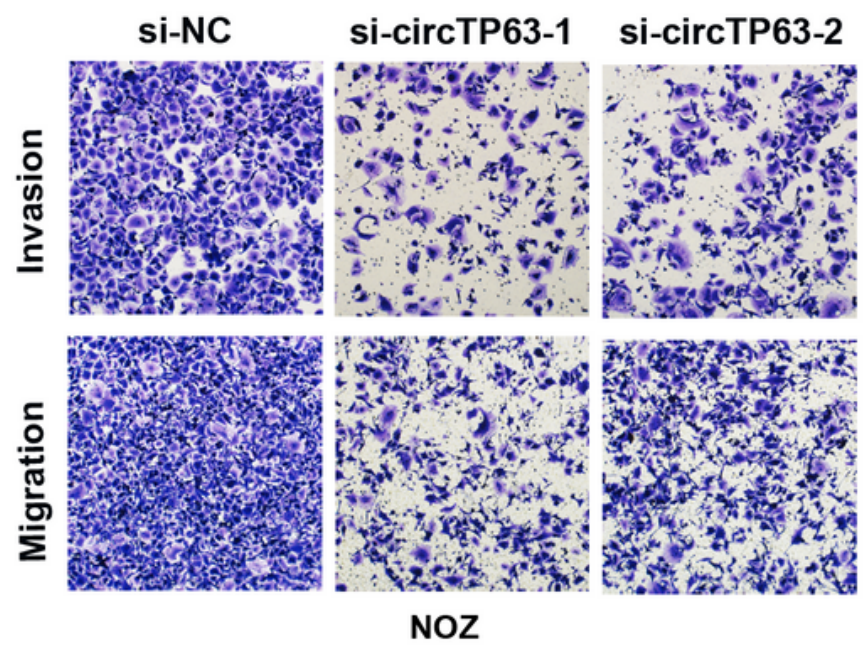

C

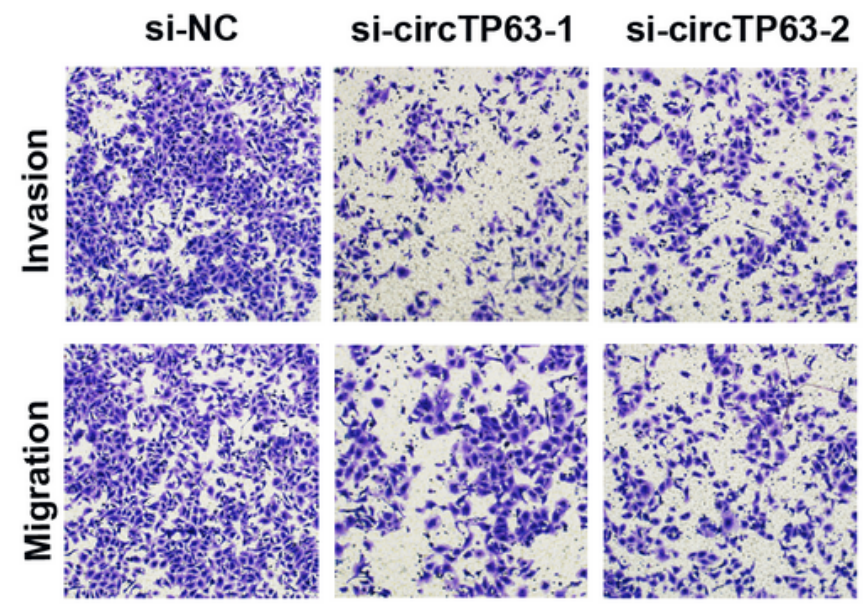

SGC-996

E

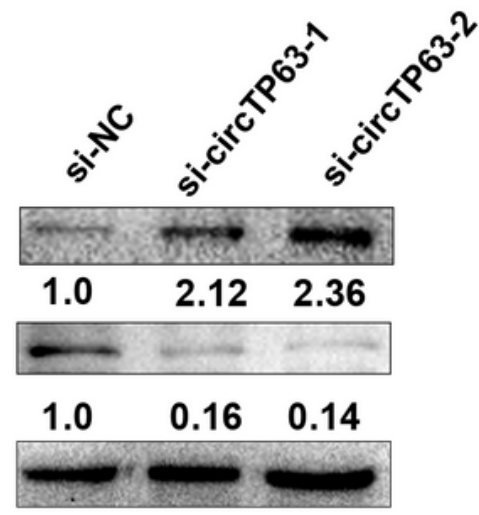

NOZ

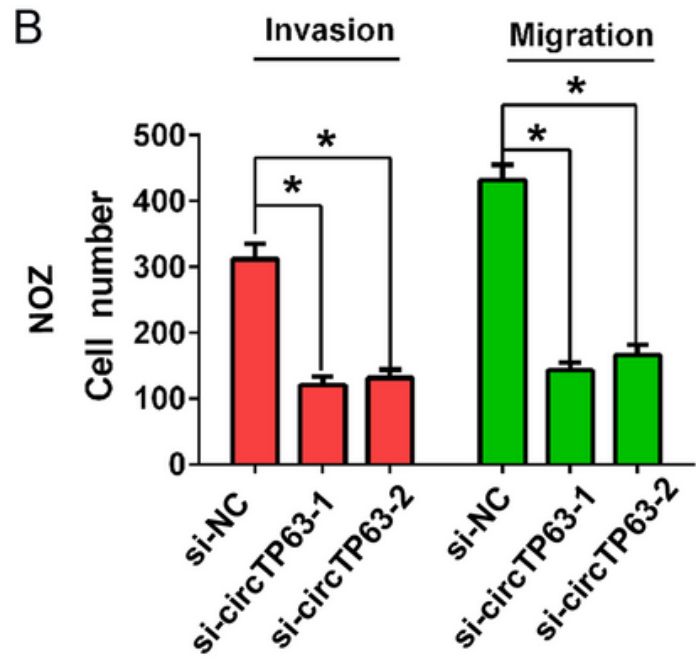

D

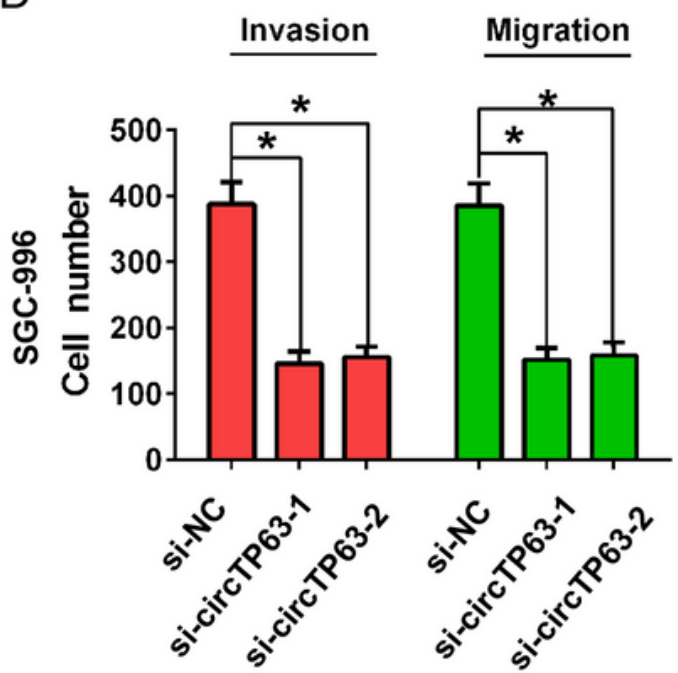

F

E-cadherin

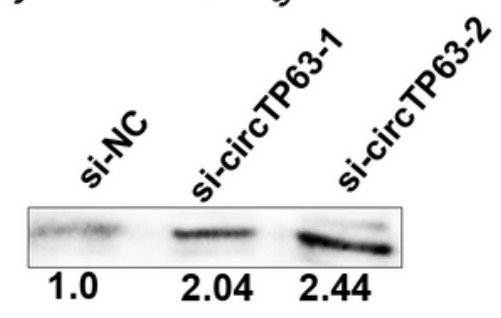

Vimentin

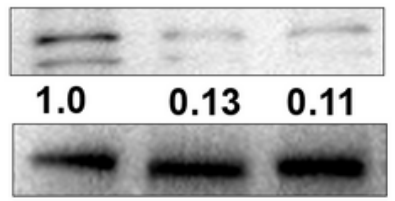

SGC-996

Figure 3

CircTP63 knockdown suppressed cell migration, invasion and cell EMT progression in GBC. (A)-(B) The cell migration and invasion abilities were analyzed by transwell assays after cells were transfected with si-NC, si-circTP63-1 or si-circTP63-2 in NOZ cells. (C)-(D) The cell migration and invasion abilities were analyzed by transwell assays after cells were transfected with si-NC, si-circTP63-1 or si-circTP63-2 in SGC-996 cells. (E)-(F) The protein expression of E-cadherin or Vimentin was analyzed by western blot 
analysis after cells were transfected with si-NC, si-circTP63-1 or si-circTP63-2 in NOZ or SGC-996 cells. Data are shown as mean \pm S.E.M., ${ }^{*} P<0.05$.

A

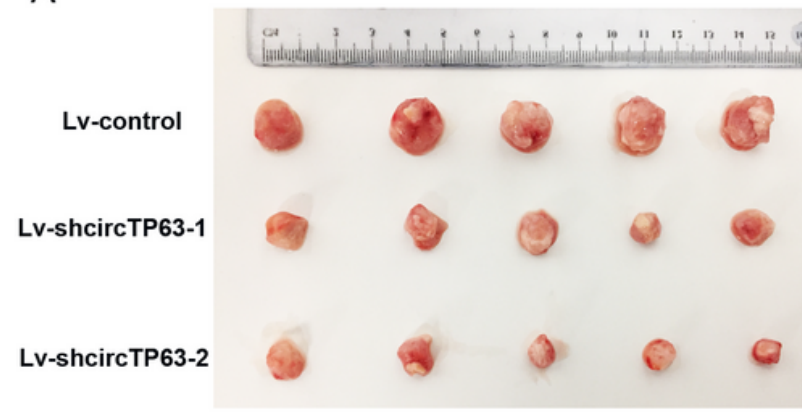

B

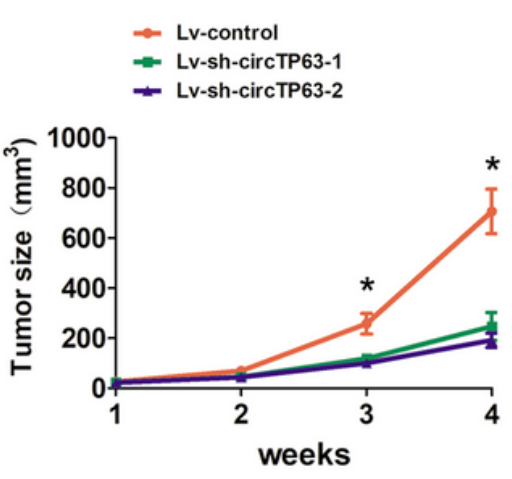

C

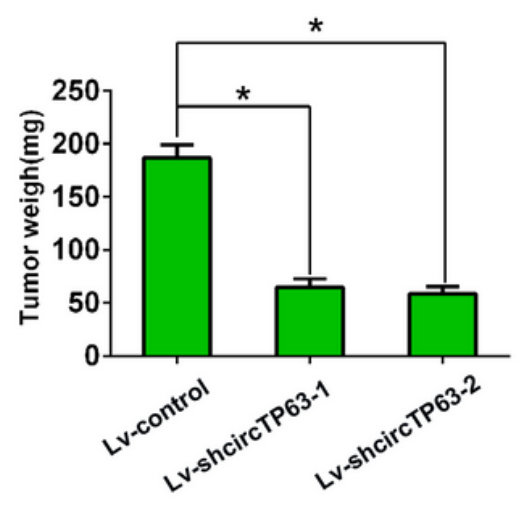

D

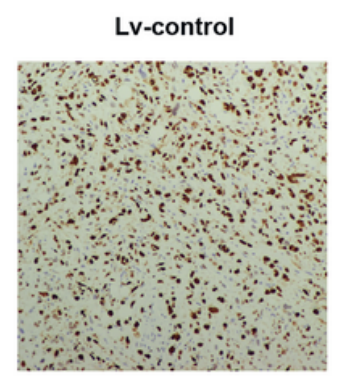

Lv-shcircTP63-1

Lv-shcircTP63-2
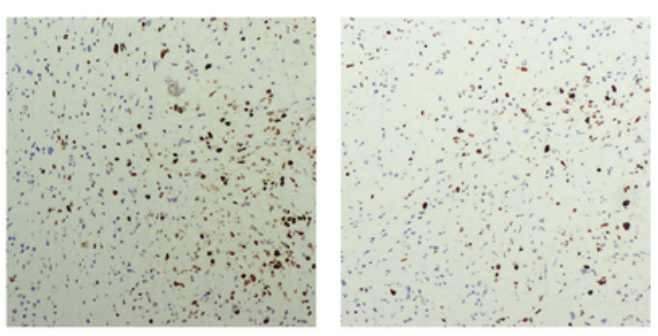

\section{Figure 4}

CircTP63 knockdown suppressed cell growth in GBC in vivo. (A) Representative images of subcutaneous xenograft tumors ( $n=5$ for each group). (B) Growth curves of tumor volume, which were measured every week. (C) Growth curves of tumor weight, which were measured every week. (D) The protein levels of Ki67 were analyzed based on IHC staining. ${ }^{*} \mathrm{P}<0.05$. 
A
cirTP63-WT 5'-GAGUCCCUGGAACUCAUGCAGUA-3'
miR-217 3'-AGGUUAGUCAAGGACUACGUCAU-5'
cirTP63-MUT 5'-GAGUCCCUGGAACUCUACFUCAA-3'

D

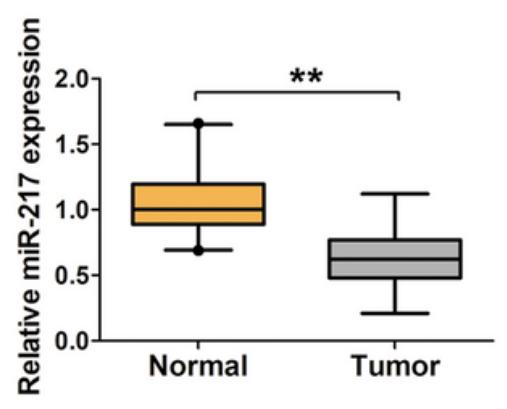

B

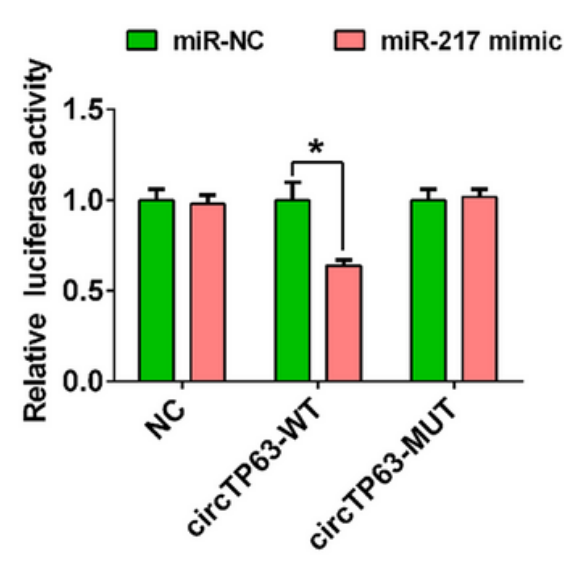

E

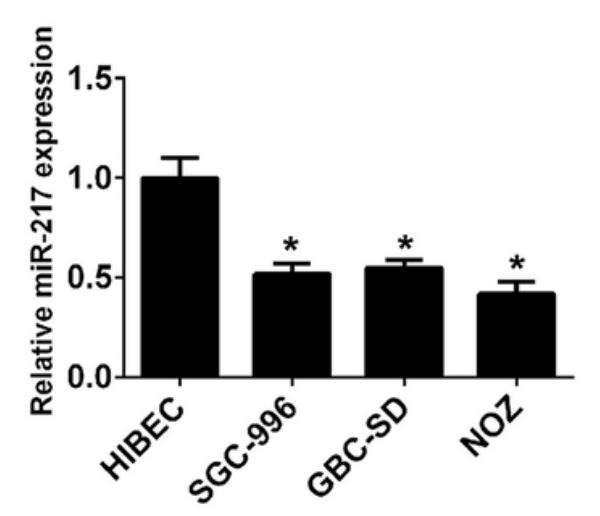

C

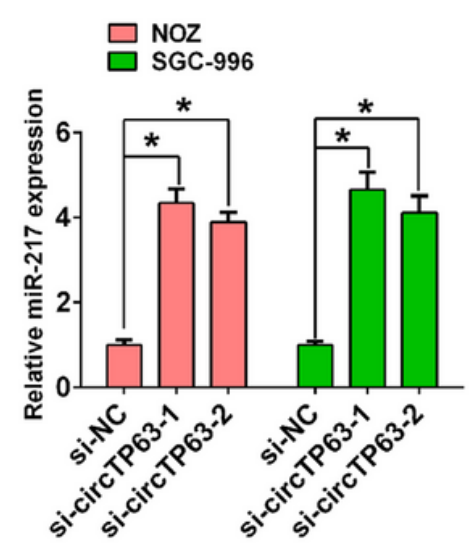

$\mathrm{F}$

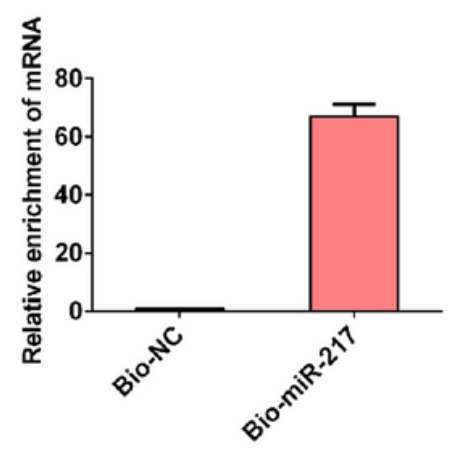

Figure 5

CircTP63 functions as a sponge of miR-217. (A) schematic of the wild-type (WT) or mutant (MUT) circTP63 luciferase reporter vectors was constructed. (B) The luciferase activities of the circTP63-WT or circTP63-MUT luciferase reporter vector in NOZ cells transfected with miR-217 mimic or NC. (C) The relative expression of miR-217 was detected after NOZ or SGC-996 cells were transfected with si-NC, sicircTP63-1 or si-circTP63-2 by qRT-PCR analysis. (D) The relative expression of miR-217 was detected in GBC tissues and adjacent normal tissues by qRT-PCR analysis. (E) The relative expression of miR-217 was detected in three GBC cell lines (NOZ, SGC-996 and GBC) and the human intrahepatic biliary epithelial cell line HIBEC by qRT-PCR analysis. (F) The bio-miR-217 or NC group complex was pulled down by incubating the cell lysate with streptavidin-coated magnetic beads and the circTP63 was detected by qRT-PCR. Data are shown as mean \pm S.E.M., ${ }^{\star} \mathrm{P}<0.05$. 
A

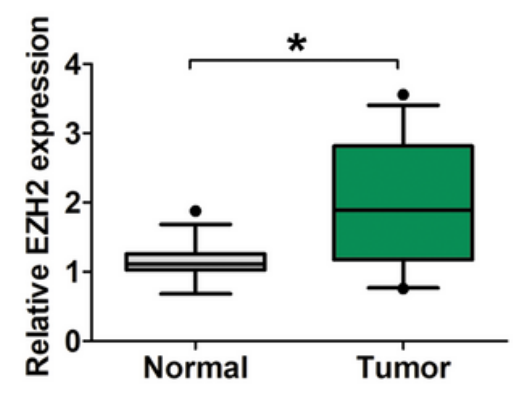

B

miR-217 3'-AGGUUAGUCAAGGACUACGUCAU-5' EZH2-WT 5'-UGAACUUUAUAAAAAGCAAUGCAGUA-3'

EZH2-MUT 5'-UGAACUUUAUAAAAAGCAUACGUCAA-3'

C

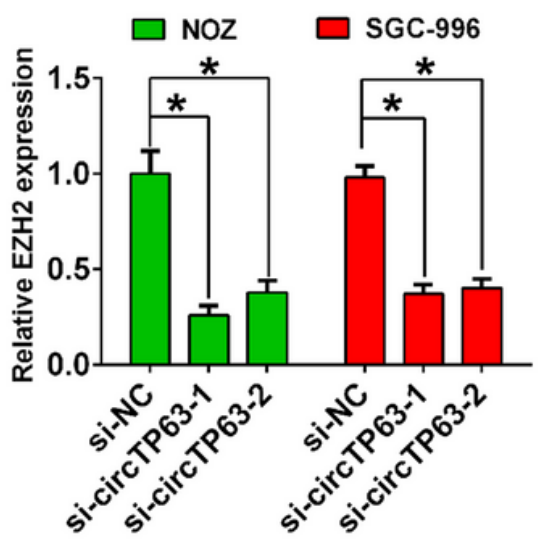

E

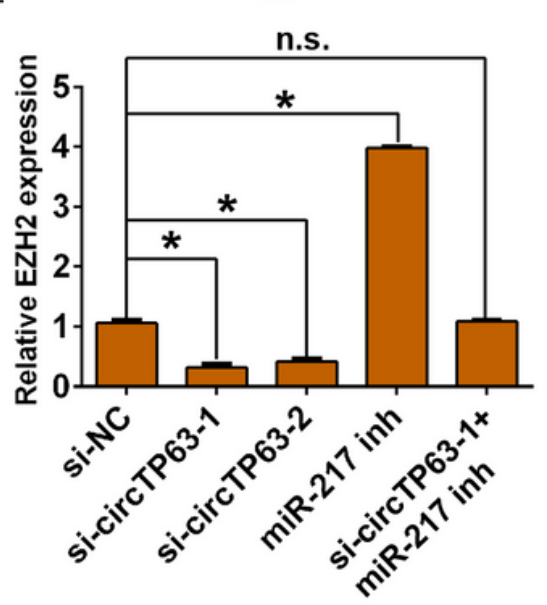

$\mathrm{F}$

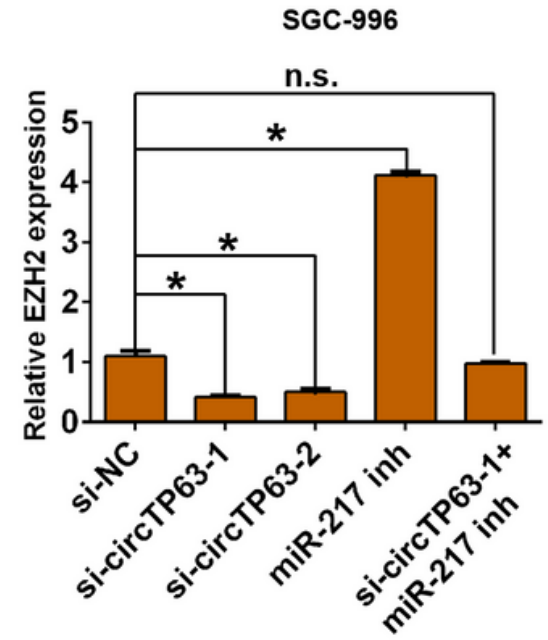

G

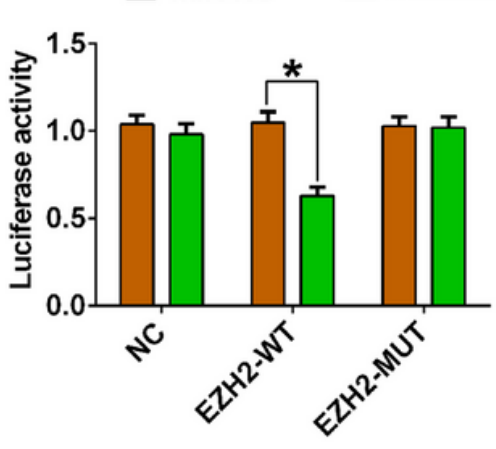

NOZ

$\mathrm{H}$

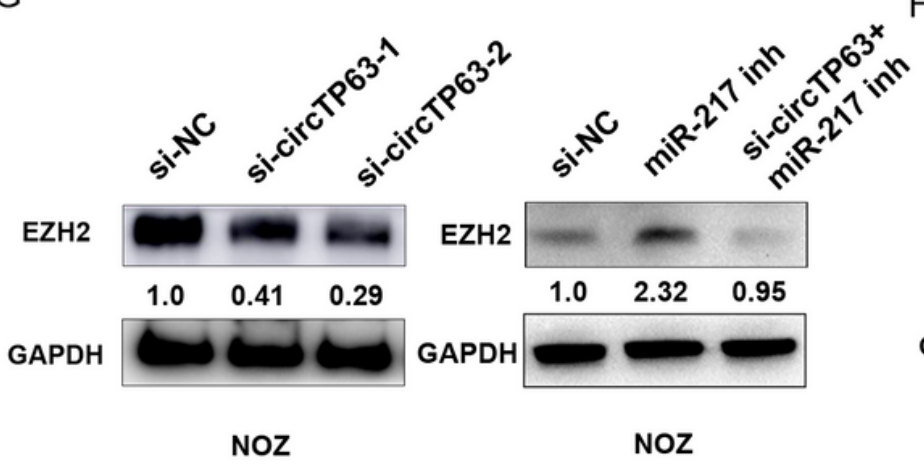

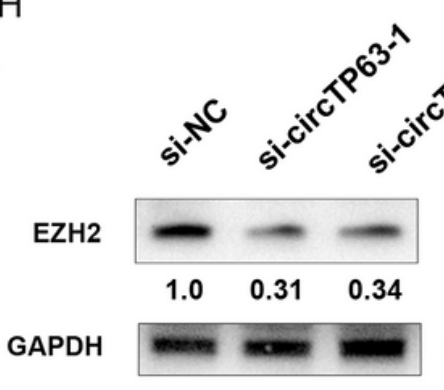

SGC-996

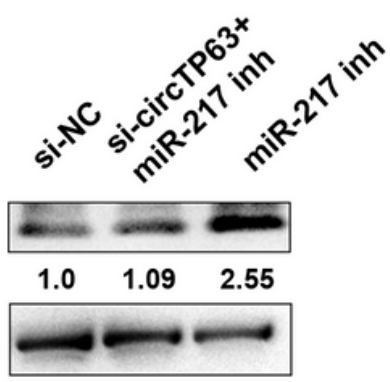

SGC-996

\section{Figure 6}

EZH2 is a downstream target of miR-217 and is indirectly regulated by circTP63. (A) The relative expression of EZH2 was detected in GBC tissues and adjacent normal tissues by qRT-PCR analysis. (B) Schematic of the wild-type (WT) or mutant (MUT) EZH2 luciferase reporter vectors was constructed. (C) The relative expression of EZH2 was detected in after NOZ or SGC-996 cells were transfected with si-NC, si-circTP63-1 or si-circTP63-2 by qRT-PCR analysis. (D) The luciferase activities of the EZH2-WT or EZH2MUT luciferase reporter vector in NOZ cells transfected with miR-217 mimic or NC. (E)-(F) The relative expression of EZH2 was detected in after NOZ or SGC-996 cells were transfected with si-NC, si-circTP63- 
1, si-circTP63-2, miR-217 inhibitor and si-circTP63-1+miR-217 inhibitor by qRT-PCR analysis. (G)-(H) The relative protein expression of EZH2 was detected in after NOZ or SGC-996 cells were transfected with siNC, si-circTP63-1, si-circTP63-2, miR-217 inhibitor and si-circTP63-1+miR-217 inhibitor by western blot analysis, respectively. Data are shown as mean \pm S.E.M., ${ }^{*} P<0.05$. 